\title{
THE METAL ABUNDANCE OF METAL-RICH GLOBULAR CLUSTERS
}

\author{
Raffaele Gratton \\ Astronomical Observatory of Rome \\ Maria Lucia Quarta \\ Institute of Space Physics, Frascati \\ Institute of Astronomy and Geophysics, Sao Paulo \\ Sergio Ortolani \\ Astrophysical Observatory, Asiago
}

\begin{abstract}
We think that it is possible to find the correct scale of abundance for metal-rich globular clusters thanks to the new generation of spectrographs, equipped with CCD cameras. We analyzed giants in ten globular clusters and Arcturus using high dispersion spectra acquired through the CASPEC spectrograph at the $3.6 \mathrm{~m}$ telescope at La Silla. The detector was an RCA CCD. Stars cooler than $4150 \mathrm{~K}$ were avoided since their absorption spectrum is too strong. By a comparison with standard Arcturus spectra, we found a small trend to overestimate equivalent widths. This systematic error affects the derived abundances only marginally. However, too large equivalent widths must produce too large metal abundances. Abundances were derived following a standard procedure.
\end{abstract}

Since line analysis may be affected by systematic errors due to continuum tracing, we derived abundances also by a comparison with synthetic spectra, using a technique which avoids any continuum tracing. This comparison provides a weighted mean abundance of metals biased versus Fe. A total error of 0.15 dex may be given to the abundances derived from synthetic spectra. The derived abundances are presented in Table I. Line analysis probably overestimates the true metal abundance (due to the use of equivalent widths which are too large). Synthetic spectra probably underestimate the abundances (due to a possible zero point error in wavelengths). Synthetic spectra are completely independnet of continuum tracing. The agreement between line analysis and synthetic spectra gives us confidence in our abundances for $[\mathrm{Fe} / \mathrm{H}]<-1.5$. Synthetic spectra are inaccurate for very low metal abundance since the selected spectral region includes only quite weak lines. The comparison with previous high dispersion abundance determinations (Pilachowski et a1., 1983, PSW) is quite poor. $<[\mathrm{Fe} / \mathrm{H}] \mathrm{LA}-[\mathrm{Fe} / \mathrm{H}] \mathrm{PSW}\rangle=+0.02 \pm 0.11(\sigma=0.30)$. The comparison with

495

J. E. Grindlay and A. G. Davis Philip (eds.),

The Harlow-Shapley Symposium on Globular Cluster Systems in Galaxies, 495-496.

(C) 1988 by the IAU. 
other metal-abundance determinations (from photometric indices and low dispersion spectroscopy) is good.

Oxygen abundances were derived by means of synthetic spectra of the region including the $6300.31 \AA$ [OI] line. The use of relatively hot stars makes CO formation of less importance than in previous studies. Errors are about $0.2 \mathrm{dex}$. The comparison between our oxygen abundances and those by PSW is poor $\langle[0 / \mathrm{H}] \mathrm{US}-[0 / \mathrm{H}] \mathrm{PSW})\rangle=+0.22 \pm 0.13$ $(\sigma=0.32)$. The comparison with $\mathrm{R}(\mathrm{CO})$ (Caputo 1985) is good. If $0>\mathrm{C}$, the correlation of $\mathrm{R}(\mathrm{CO})$ with oxygen abundances indicates that carbon and oxygen abundances are correlated. The cluster to cluster scatter in $[0 / \mathrm{Fe}]$ is within the uncertainties in the analysis. There is no evidence for an underabundance of oxygen for NGC 288, while NGC 6752 provided a low oxygen abundance (significant at a $2 \sigma$ level). In the mean, they do not differ from the other clusters. The mean oxygen overabundance in the observed globular clusters is $\langle[0 / \mathrm{Fe}]\rangle=+0.4$ \pm 0.1 . Oxygen is overabundant as in field halo stars (see e.g. Sneden et al. 1979). $\alpha$-elements ( $\mathrm{Mg}, \mathrm{Si}, \mathrm{Ca}$ and $\mathrm{Ti}$ ) are overabundant by $\langle[\alpha / \mathrm{Fe}]\rangle=+0.3 \pm 0.1$. From our abundances, the metal enrichment process of globular cluster material is indistinguishable from that of halo stars. The simplest interpretation is that there was no important self pollution.

\section{REFERENCES}

Caputo, F. 1985 Astron. Astrophys. 147, 317.

Pilachowski, C. A., Sneden, C., and Wallerstein, G. 1983 Astrophys. J. Suppl. 52, 241 (PSW).

Sneden, C., Lambert, D. L., and Whitaker, R. W. 1979 Astrophys. J. 231,762 .

\section{TABLE I}

Abundances

\begin{tabular}{|lcccc|}
\hline Cluster & {$[\mathrm{Fe} / \mathrm{H}] \mathrm{LA}$} & {$[\alpha / \mathrm{Fe}]$} & {$[\mathrm{Fe} / \mathrm{H}] \mathrm{SS}$} & {$[\mathrm{O} / \mathrm{H}]$} \\
\hline Arcturus & $-0.54+0.04$ & $+0.20+0.09$ & $-0.47+0.05$ & $-0.34+0.07$ \\
47 Tuc & $-0.82+0.03$ & $+0.18+0.08$ & $-0.88+0.08$ & $\ldots$ \\
NGC 288 & $-1.31+0.03$ & $+0.34+0.08$ & $-1.35+0.09$ & $-0.76+0.07$ \\
NGC 362 & $-1.18+0.03$ & $+0.22+0.05$ & $-1.10+0.09$ & $-0.96+0.18$ \\
NGC 5897 & $-1.84+0.01$ & $+0.22+0.04$ & $-2.36+0.16$ & $-1.44+0.11$ \\
M5 & $-1.42+0.05$ & $+0.29+0.04$ & $-1.44+0.04$ & $-0.93+0.05$ \\
M4 & $-1.32+0.03$ & $+0.36+0.13$ & $-1.48+0.04$ & $-0.99+0.06$ \\
NGC 6352 & $-0.79+0.06$ & $+0.23+0.06$ & $-0.75+0.04$ & $-0.49+0.04$ \\
NGC 6362 & $-1.04+0.06$ & $+0.33+0.06$ & $-1.05+0.07$ & $\ldots$ \\
NGC 6752 & $-1.53+0.05$ & $+0.31+0.06$ & $-1.68+0.06$ & $-1.37+0.09$ \\
M71 & $-0.81+0.04$ & $+0.28+0.05$ & $-0.89+0.04$ & $-0.16+0.06$ \\
\hline
\end{tabular}

